\title{
Memory for language organization in concrete and abstract sentences
}

\author{
SHANNON DAWN MOESER \\ Memorial University of Newfoundland, St. John's, Newfoundland, Canada
}

\begin{abstract}
Subjects were presented with concrete and abstract sentences equal in lexical complexity and comprehension rating and tested on their ability (1) to recognize the words used in the sentences when presented at a 3 -sec rate and (2) to recreate the initial sentences when presented with a list of the words used. Only on this second task was there a significant concrete/abstract difference. In a second experiment, there was a significant concrete/abstract difference in word recognition when a 6-sec decoding rate was used. It was suggested that the best explanation of these results could be offered by a semantic analog model of language memory.
\end{abstract}

This paper is concerned with the effect of verbal concreteness on the storage of sentence information. Paivio (1971) has proposed that verbal material is encoded into two representational codes, imaginal and verbal, which differ in their organizational properties, the imaginal code being specialized for integration of stimulus information into a unitized structure and the verbal code being specialized for sequential organization of elements. According to Paivio's dual-coding theory, concrete verbal material is stored in both codes whereas abstract verbal material is primarily stored in the verbal code. In support of the dual-coding model, Begg and Paivio (1969) reported that subjects presented with concrete sentences were superior to those piesented with abstract sentences in recognizing changes in stimulus item organization (subject-object reversals) whereas those presented with abstract sentences were superior to those presented with concrete sentences in recognizing element changes (synonym substitutions). Johnson, Bransford, Nyberg and Cleary (1972) have criticized the Begg and Paivio study on the basis that the concrete and abstract sentences used in the study were not equal in comprehensibility nor in amount of change effected by the subject-object reversals. Moeser (1974) found that when these factors were controlled, subjects were still superior at recognizing organizational changes in concrete sentences. However, Moeser also found that her subjects were superior at recognizing element changes in abstract sentences only under very limited conditions which probably could be attributed to response bias mechanisms. Under most conditions, element changes were more easily recognized in concrete sentences.

Moeser (1974) argued that her data gave more support to a semantic analog model of sentence memory storage than to a dual-coding interpretation. In terms of

This work was supported by National Research Council of Canada Grant A9638 to the author. both analyses of latency data and data on the type of change most easily identified, it appeared that both concrete and abstract sentences were being encoded into a semantic representation, but that concrete sentences were more easily encoded into this system. The superiority of concrete sentences in memory could be explained by assuming that the structure of the semantic code was based on perceptual organizational principles. Thus the concrete sentences, which were easily translated into a perceptual code, were easy to represent as a complete structure in memory. The abstract sentences, which involved difficult perceptual transformations, were likely to be represented as several structural configurations, each incorporating portions of the verbal sequence. The difficulty of representing abstract sentences as a holistic structure resulted in a poorer identification of both meaning and wording changes in abstract sentences.

This argument is similar to Begg's (1972) proposal that concrete verbal material is more redintegrative than abstract material. Begg found that, with concrete phrases, the recall of one part highly facilitated the recall of the other part, but that this was not true of abstract phrases. Begg (1973) pointed out the resemblance of his data when using concrete and abstract verbal materials and when using integrated and nonintegrated imagery instructions. In his experiments, subjects exposed to concrete material and those instructed to form integrated images of concrete material showed superior redintegrative memory to subjects exposed to abstract material or instructed to form nonintegrated images of concrete material. Similarly, in a recently completed study by the author, the results of the Moeser (1974) experiment were duplicated using concrete sentences with interactive and noninteractive imagery instructions in place of concrete and abstract sentences. Thus it might be concluded that the difference between the storage of concrete and the storage of abstract sentences could be attributed solely to the superior integrative 
properties of concrete sentences. The present set of experiments was undertaken to investigate this hypothesis.

\section{EXPERIMENT I}

Experiment I was designed to answer two questions. The first concerned the effect that different encoding instructions had on the processing of sentences and their separate words. Moeser (1974) suggested that, when subjects are asked to "comprehend" a sentence, they are often likely to judge the sentence as being understood as soon as they understand the meanings of the individual words which comprise the sentence; for this reason, sentences which have the same comprehension rating may still be remembered differently if they differ in terms of the ease with which they can be formed into an integrated representation. To investigate this hypothesis, subjects were asked to look at a sentence until they comprehended it; later they were tested on their ability to remember the individual words which had appeared in the sentence and to remember the way in which these individual words were organized. The results of these tests were compared with the same tests given to subjects who were told that they were going to be given a memory test following the presentation of the sentences and to subjects who were required to add a descriptive phrase to the sentences which required an understanding of their holistic meaning. Thus three encoding instruction conditions were used in Experiment I. Subjects were asked to comprehend the sentence, to remember the sentence for a later test, or to add a descriptive phrase to the sentence.

The second question investigated in this experiment was whether the major difference between the recall of concrete and abstract sentences could be attributed to integrative processing or whether the concrete and abstract words which occured in the sentences were also encoded differentially. In an unpublished study by Ted Rowe at Memorial University, approximately four times as many words were recalled from concrete sentences as from abstract sentences, and Begg (1972) reported that the words presented as part of concrete phrases were recalled approximately twice as often as the words presented as parts of abstract phrases. On the other hand, Bower (1970) found that integrative imagery instructions did not facilitate recognition memory for individual words used in the experiment; likewise, Begg (1973) found that integrative imagery instructions did not facilitate memory in a noncued recall task. Thus it may be that concrete material is not only more easily integrated than abstract material but also shows superior coding of the individual elements. Such a finding would support Paivio's (1971) dual-coding theory. However, it may be that the poorer recall of abstract elements was due to the poorer integrative properties of abstract material. Thus if the possibility of redintegration is minimized there may not be differences in the recognition of concrete and abstract elements.

Therefore, Experiment I used two recognition tests. In the first test, subjects listened to a series of individual words, some of which had appeared in the sentences and some of which had not. The words were presented in a random order at a speed too fast to redintegrate the entire sentence, and subjects were required to indicate which words had been part of the sentences and which had not. In the second test, a list of all the words used in the sentences was given to the subjects and they were required to combine these words to form the sentences they had seen. Thus if the major difference in memory for concrete and abstract sentences is due to the integrative processing, the concreteness dimension should have a significant effect on the sentence formation test but not on the word recognition test. If the concreteness dimension affects both redintegrative memory and word storage memory, both tests should show a significant concrete/abstract difference. And, if the major effect of concreteness is on word storage, the concrete/abstract difference should only be significant on the word recognition test.

\section{Methods}

Sentences. Ten concrete and ten abstract sentences were constructed using the syntactical frame "The (adjective) (noun) (verb) a (adjective) (noun)." These sentences were part of a set of sentences which had been rated by a group of 55 paid volunteers from undergraduate psychology classes at Memorial University of Newfoundland on a seven-point scale from "difficult to understand" to "easy to understand." The sets of concrete and abstract sentences used in the present experiment were matched in terms of this comprehensibility rating and in terms of lexical complexity.

Apparatus. The sentences were presented on $35-\mathrm{mm}$ slides projected by a Kodak 800 projector equipped with a Gerbrands shutter and Control Model G1 165 connected to a 5304 timer, a H/P 5055A digital recorder, and a H/P 5300A measuring system, and controlled by a hand-operated button switch. When the projector was switched on, the shutter opened and the timer began operating. When the button switch was pressed, the shutter closed, the timer stopped, and the printer printed out the time between the opening and closing of the shutter; then the projector advanced to the next slide and the shutter opened again. The time between the closing and opening of the shutter was 1.8 sec. The cycle repeated each time the button switch was pressed.

A Uher 4000 Report-L tape recorder was used for the word recognition test, with a tape speed of $7 \frac{1}{2}$ ips and with earphones attached.

Subjects. Thirty-six undergraduate paid volunteers attending classes at Memorial University of Newfoundland were tested. Twelve subjects (six male and six female) were assigned to each of the instruction group conditions. As two separate sets of five concrete and five abstract sentences were presented, three male and three female subjects in each instruction condition received each set of sentences.

Procedure. Subjects were tested individually. Each was shown how to operate the switch that changed the slides and was told that he could look at each slide as long as he wished. Subjects in the comprehension instruction group were told to change the slide "as soon as they understood the meaning of the sentence." Subjects in the memory instruction group were told that they 
would be given "a memory test" after they had seen the slides. Subjects in the elaboration instruction group were told to complete each sentence with a "because" phrase which explained the meaning of the sentence.

Two sets of sentences were used, with five concrete and five abstract sentences in each set. For both sets, the concrete and abstract sentences were presented in alternating order, with a concrete sentence being presented first in one set and an abstract sentence being presented first in the other set.

After the ten sentences had been presented, subjects were given the word recognition test. One hundred words were presented, consisting of the 50 words which had appeared in the ten sentences and 50 distractor words. There were 25 concrete and 25 abstract distractor words, chosen so that none was either phonemically similar nor semantically similar to the target words. The words appeared in random order except that the concrete and abstract words were alternated. They were presented on a Uher tape recorder at the rate of one word every $3 \mathrm{sec}$ and the list numbers also appeared on the tape (i.e., Number 1, juggler; Number 2, report; Number 3, automobile, etc.). Subjects recorded their answers on an answer sheet by circling either "yes" if they thought the word had appeared in a sentence or "no" if they thought that it had not been in a sentence.

Then the subjects were given a list of the 50 words which had appeared in the sentences, arranged in alphabetical order, and were asked to try to form the sentences which had initially been presented using these words. They were allowed as much time as they wished to complete this task.

\section{Results And Discussion}

The mean concrete and abstract encoding latencies, word recognition scores, and sentence association scores for each of the three instruction groups are shown in Table I. As all of the statistical analyses showed no significant differences between the two sets of sentences, the scores for both sets have been added together in the table.

The word recognition score for each subject was computed by giving one point for each "yes" response to a word which had been presented in the sentences. The maximum possible score was 25 , with a chance score of 12.5. The sentence association score was computed by giving one point for each two words which were correctly placed in the same sentence. These two words did not have to be sequentially associated to be scored as correct. For instance, for one sentence the subject may have written down the sentence subject and the sentence object, with blanks left for the missing words, or he may have written down a five-word sequence but only the sentence subject and sentence object had appeared together in the initially presented sentences. In both cases, one point would be given for the sentence. As there were five words in each sentence, the maximum possible sentence association score was four.

All of the analyses of variance compared the three instruction groups, the concrete/abstract dimension, and the two sets of sentences on the various measures. In discussing the results, the author has accorded with Clark's (1973) argument that language should be treated as a random effect in statistical analyses. Thus a result is not accepted as statistically significant unless it can be generalized both to a different sample of subjects and to a different sample of language material; such a result is obtained only when the quasi- $F$ analysis is significant. However, results significant by subjects (treating language as a fixed effect) or by language (treating subjects as a fixed effect) will be reported.

Because of the skewedness of the latency data, reciprocals were used in analyzing the results. There was a significant difference among instruction groups $\left[F^{\prime}(2 / 34)=9.57, p<.001\right]$. The difference between the concrete and abstract sentences was significant by subjects $\left[F^{1}(1 / 16)=5.34, p<.05\right]$ and by sentences $\left[\mathrm{F}^{2}(1 / 30)=5.91 . \mathrm{p}<.05\right]$ but the quasi-F analysis was not significant. No other significant main effect or interaction was found.

With regard to the word recognition scores, none of the quasi-F comparisons were significant, although the difference between the concrete and abstract words was significant with regard to subjects $\left[\mathrm{F}^{1}(1 / 33)=5.00\right.$, $\mathrm{p}<.05]$. No other comparison showed significance. It was possible that a response bias could have affected the word recognition score, such that, for instance, subjects might have had a greater tendency to say "yes" to abstract words in general. Thus a second analysis of variance was done on the word recognition scores, this time using the total correct response score to both target and distractor words. Again, there were no significant results except for a significant concrete/abstract comparison with regard to subjects $\left[\mathrm{F}^{1}(1 / 33)=15.05, \mathrm{p}<.001\right]$.

On the analysis of the sentence association scores, the difference between instruction groups was significant $\left[F^{\prime}(2 / 37)=3.93, p<.05\right]$ and the difference between concrete and abstract sentences was significant $\left[F^{\prime}(1 / 26)\right.$ $=14.86, p<.001]$. No other significant main effects or interactions were found.

Thus the different instructions had two main effects, first, on the amount of time subjects spent encoding the

Table 1

Mean Encoding Latencies, Word Recognition Scores, and Sentence Association Scores Obtained by Subjects in Each Instructional Condition in Experiment 1

\begin{tabular}{|c|c|c|c|c|c|c|}
\hline & \multicolumn{3}{|c|}{ Concrete } & \multicolumn{3}{|c|}{ Abstract } \\
\hline & Latencies & Words & Sentences & Latencies & Words & Sentences \\
\hline $\begin{array}{l}\text { Comprehension } \\
\text { Memory } \\
\text { Elaboration }\end{array}$ & $\begin{array}{r}3.77 \\
14.16 \\
12.48\end{array}$ & $\begin{array}{l}16.75 \\
18.93 \\
17.83\end{array}$ & $\begin{array}{l}1.63 \\
2.82 \\
2.85\end{array}$ & $\begin{array}{r}4.23 \\
14.21 \\
20.26\end{array}$ & $\begin{array}{l}16.38 \\
17.08 \\
16.08\end{array}$ & $\begin{array}{r}.85 \\
1.28 \\
1.73\end{array}$ \\
\hline Mean & 10.14 & 17.86 & 2.43 & 12.90 & 16.50 & 1.29 \\
\hline
\end{tabular}


sentences, and second, on the subjects' ability to associate the words into sentences. The subjects given the comprehension instructions had significantly faster encoding latencies and were significantly poorer on the sentence association task than the subjects in the other two instructional groups. As there were no significant interactions between instruction condition and the sentence concreteness, the instructions had basically the same effects on both types of sentences.

The different instructions and consequently longer encoding latencies did not significantly affect the subjects' ability to recognize the words which had appeared in the sentences. This result supports the Moeser (1974) suggestion that subjects often judge a sentence as "comprehended" when they understand the meanings of the individual words in the sentence. Subjects asked to comprehend the sentence seemed to have encoded the individual words as effectively as subjects asked to remember the sentence or asked to elaborate on the sentence. In the memory group, subjects did not know what type of memory test they would receive and it can be assumed that they would try to remember as much as they could about the sentences, including the wording. In the elaboration group, subjects were encouraged to relate to the meaning of the sentence as a whole, rather than attend to the meanings of individual words. Yet all three groups showed roughly the same mean word recognition score. Thus it appears that encoding the words into some form of memory system is an important part of encoding the sentence as a whole.

The difference between the concrete and abstract verbal material was significant using the quasi-F statistic only with regard to the association of words into sentences. The major effect of concreteness seemed to be in terms of organization. The subjects remembered the connections between words in the concrete sentences far better than in the abstract sentences.

The encoding latency results showed a significant concrete/abstract effect with regard to words and to sentences but the quasi-F statistic was not significant. In all of the Moeser (1974) studies, it also took subjects longer to encode abstract sentences than to encode concrete sentences even when the two types of sentences were equated in terms of comprehension rating and lexical complexity. In some of these experiments the quasi-F statistic was significant; in all of them the effect was significant by subjects.

\section{EXPERIMENT II}

In Experiment $\mathrm{I}$ the difference between recognition of the concrete words and recognition of the abstract words was not significant when the words were treated as a random variable. Likewise, when Bower (1970) contrasted interactive imagery instructions with non- interactive imagery instructions, he failed to find any significant difference between the two conditions in terms of recognition memory for words, although he found a strong effect with respect to associative memory. However, when Peterson and Murray (1973) compared the differential effects of imagery instructions, they did obtain a positive effect of interactive imagery on the recognition of words. Also, in Experiment $I$ of the present paper, the recognition of concrete words was superior to the recognition of abstract words, and this difference was significant by subjects if not by words. The concrete/abstract difference was greatest in those instruction conditions in which longer encoding instructions occurred. Thus it may be that under certain conditions a significant recognition difference can be obtained.

However, it may also be that when significant recognition differences are obtained, the results can better be explained in terms of a redintegration hypothesis rather than in terms of differential encoding mechanisms. Peterson and Murray (1973) allowed longer times for stimulus recognition than did Bower (1970). In Experiment I, a fast presentation speed was used in order to preclude the possibility that the subjects would redintegrate the entire sentence and thus make their judgments in terms of associational structure rather than in terms of the absolute encoding of the word. Yet the $3-\mathrm{sec}$ period was not so short that it would impede the arousal of images to the concrete words; Paivio (1966) obtained a mean latency of about $2.5 \mathrm{sec}$ for image arousal to concrete words.

Experiment II was designed to test whe ther significant concrete/abstract word recognition differences could be obtained when a longer recognition time was allowed. It may be that a positive effect of integrative organization on stimulus recognition occurs when time is allowed for the associational structure to be remembered. In other words, the subjects' judgments may sometimes be made in terms of what they can remember about the sentence or phrase in which the word occured as well as in terms of their absolute encoding of the word.

\section{Method}

The sentences, apparatus, and materials used in Experiment II were identical to those used in Experiment I. The same words and word order were used in the word recognition test as were used in Experiment 1 , but the tape itself was re-recorded using a female assistant who was ignorant of the purpose of the experiment. She recorded the two word recognition tests used in Experiment $I$ at the rate of one word every $3 \mathrm{sec}$ and the same two tests at the rate of one word every $6 \mathrm{sec}$. As in the earlier experiment, the list numbers were recorded with the words.

Imagery control. A control experiment was run to see if subjects could indeed form images to the words as they occurred on the tape. Twenty-four undergraduate students were used for this imagery control. They were tested individually with six (three male and three female) listening to each test. These subjects were never exposed to the sentences but were simply instructed to listen to the tape and circle "yes" if they could form an image to the word before the next word occurred and 
"no" if they could not form an image to the word in the time allotted. The results showed that, at the $3-\mathrm{sec}$ rate, $89 \%$ of the target concrete words were imagable within the time period ( $86 \%$ of both the target and distractor concrete words) and $56 \%$ of the target abstract words were rated as imagable (55\% of both target and distractor abstract words). At the $6-\mathrm{sec}$ rate, $94 \%$ of the target concrete words $(91 \%$ of both target and distractor concrete words) and $85 \%$ of the target abstract words ( $86 \%$ of the target and distractor abstract words) were imagable. Thus it does not appear that insufficient time to make use of the imagery code could be used as an explanation of the results.

Subjects. Twenty-four subjects were used, twelve in each group. Three female and three male subjects heard each set of words in each group. All subjects were undergraduate students attending Memorial University.

Procedure. The procedure used was similar to the procedure used in Experiment I except that all subjects were told that they would receive a memory test "on the sentences" after they had seen the slides. After the subject had seen the 10 sentences he was given one of the word recognition tests.

\section{Results And Discussion}

The mean concrete and abstract encoding latencies and word recognition scores for both the 3 -sec group and the 6-sec group are shown in Table 2. The word recognition data have been broken down to show the mean number of target words correctly identified and the mean total response score to both target and distractor words. As can be seen from Table 2, for both groups, the concrete sentences were encoded faster and the concrete words were more often identified correctly.

Analyses of the two groups were performed separately. For the $3-\mathrm{sec}$ group there was no significant concrete/abstract difference in the recognition of the target words, either by subjects or by words. Nor were there any significant differences either by subjects or by words when the total scores were analyzed (that is, correct responses to both target and distractor words). The analysis of the encoding latencies in the $3-\mathrm{sec}$ group showed a significant concrete/abstract difference by subjects $\left[F^{1}(1 / 10)=5.45, p<.05\right]$ and by sentences $\left[\mathrm{F}^{2}(1 / 16)=5.72, \mathrm{p}<.05\right]$ but the quasi-F statistic was not significant.

For the 6-sec group, there was a significant concrete/abstract difference with both the target words $\left[\min F^{\prime}(1 / 30)=12.28, p<.01\right]$ and the total scores $\left[\min F^{\prime}(1 / 28)=5.97, p<.05\right]$. Analysis of the encoding latency data showed a significant concrete/abstract difference only with regard to subjects $\left[F^{1}(1 / 10)=5.25, p<.05\right]$.

In addition, analyses were made comparing the two groups. No significant Group by Concreteness interactions were found on the latency data for total recognition scores, but the interaction was significant on the target words by subjects $\left[F^{1}(1 / 22)=4.00\right]$ and by words $\left[F^{2}(1 / 1 / 98)=11.36\right]$. The quasi-F analysis was almost significant $\left[\min \mathrm{F}^{\prime}(1 / 39)=2.96, \mathrm{p}<.10\right]$. In none of the comparisons of latency data, total recognition scores, or target recognition scores was there a significant group difference.

Thus the concrete/abstract difference was only
Table 2

Mean Concrete and Abstract Encoding Latencies and Word Recognition Scores for Both the 3-Sec and the 6-Sec Group

\begin{tabular}{cccc}
\hline Latencies & $\begin{array}{c}\text { Target } \\
\text { Words }\end{array}$ & $\begin{array}{l}\text { Total } \\
\text { Score }\end{array}$ \\
\hline 3-Sec Presentation & & & \\
Concrete & 11.60 & 17.92 & 38.50 \\
Abstract & 13.91 & 16.92 & 36.08 \\
Mean & 12.76 & 17.42 & 37.29 \\
6-Sec Presentation & & & \\
Concrete & 12.24 & 19.92 & 41.50 \\
Abstract & 13.30 & 14.92 & 36.17 \\
Mean & 12.77 & 17.42 & 38.84 \\
\hline
\end{tabular}

significant on the recognition data for subjects given a 6-sec decoding period. Although there was a tendency for concrete words to be identified correctly more often in the 3-sec group, this tendency was not significant, not even by subjects as was found in Experiment I. (In Experiment I, the memory group was told that they would later be given a memory test; in Experiment II, the subjects were told that they would later be given a memory test on the sentences. This instructional difference may have had an effect on the word recognition results.) It is interesting to note that the comparison between groups on the recognition data was not significant but that the interaction effect between groups and target recognition was almost significant. When subjects were allowed $6 \mathrm{sec}$ to make a decision about a word, they seemed to get better in their recognition of concrete words and poorer in their recognition of abstract words. This result accords with a redintegration model of word recognition: When uncertain about whether or not a word has occurred, subjects will tend to accept the word as being part of the corpus if they can remember the context in which it occurred and reject it if they are unable to redintegrate the phrase or sentence which occurred with the word.

\section{EXPERIMENT III}

The results of Experiments I and II suggest that the major difference between how concrete and abstract sentences are stored in memory appears to be with regard to difficulties encountered in storing the organizational structure of abstract sentences. Experiment III was designed to test whether or not subjects could improve their organizational memory if they knew precisely what type of test was to be given. Subjects were given two practice trials with the procedure before being shown the 20 experimental sentences.

\section{Method}

Ten new concrete and 10 new abstract sentences were constructed using the same procedure as in Experiment $I$. Thus 
there was a total of 40 sentences which were divided into four sets, with each set containing five concrete and five abstract sentences. The procedure used was the same as in Experiment I except that all subjects were told that they would receive a memory test after they had seen the sentences. After the first set of ten sentences was shown, the subjects were given the sentence association test; this was repeated for the next three sets of sentences. The third and fourth sets of sentences were identical to the two sets used in Experiment I.

Subjects. Five male and five female undergraduate paid volunteers attending classes at Memorial University of Newfoundland served as subjects.

\section{Results And Discussion}

Data analyses were performed only on the last two sets of 10 sentences. These two sets contained the same sentences that were used in Experiment I. The mean concrete and abstract latencies and sentence association scores for the 10 subjects are shown in Table 3. (Sentence association scores were calculated as described in Experiment I.) As can be seen, there was little difference between concrete and abstract on the encoding latencies but considerable difference on the sentence association scores.

An analysis of variance comparing the concrete and abstract latency scores found no significant difference. However, an analysis of the sentence association scores showed a significant difference between the concrete and abstract sentences $\left[F^{\prime}(1 / 36)=14.86, p<.001\right]$.

Thus even when subjects knew what type of test they would receive they were still extremely poor at remembering the associational structure of abstract sentences.

\section{EXPERIMENT IV}

Thus far it has been argued that the difficulty of encoding the associational structure of abstract sentences in memory is related to the iconic properties of the sentence semantic structure. It is possible, however, that verbal semantic factors could be partially or wholly responsible for this difficulty. Holy oak (1974) reported that ratings of semantic relatedness could be used to account for some differences which were attributed to sentence imagability.

Perhaps the words used in the concrete sentences were more closely associated in meaning than those used in the abstract sentences. On the other hand, it may have been that the set of words used in all the abstract sentences has a higher overall associativeness than the set of words used in the concrete sentences and subjects found it more difficult to form sentences from the

Table 3

Mean Encoding Latencies and Sentence Association Scores Obtained by Subjects in Experiment III

\begin{tabular}{lrrl} 
& Concrete & Abstract & \multicolumn{1}{c}{$\mathrm{p}$} \\
\hline Latencies & 20.39 & 20.64 & n.s. \\
Sentences & 2.81 & .98 & .001 \\
\hline
\end{tabular}

abstract words because of associative interference between sentences.

To test the first hypothesis, a group of subjects was asked to rate all possible pairs of words in each sentence on an association scale. To test the second hypothesis, pairs were formed using words across sentences, and a second group of subjects was asked to rank these cross-sentence associations with respect to the associations between the words used in the sentences. Finally, a third group of subjects was asked to rate the sentences on an imagery scale to compare this rating with the results of the other two ratings.

\section{Method}

The association rating test consisted of all possible pairs of words from each of the 20 sentences used in the experiments. The words of each pair appeared side-by-side in the order in which they appeared in the sentence. Thus the subject modifier of each sentence was paired with the subject, verb, object modifier, and object; the subject was paired with the verb, object modifier, and object; the verb was paired with the object modifier and object; and the object modifier was paired with the object. As there were 10 associated pairs for each sentence and 20 sentences, a total of 200 pairs were formed in this way. Each sentence's pairs were distributed randomly throughout the whole 200 pairs, with the exception that concrete and abstract pairs were alternated. A five-point scale appeared below each pair. Subjects were instructed to circle a number indicating how closely they thought the two words were associated, with 5 indicating very low association.

In the cross-sentence ranking test, the 20 sentences were divided into four groups - the five concrete sentences from one set, the five abstract sentences from that same set, the five concrete sentences from the second set, and the five abstract sentences from the second set. For each group, five cross-sentence comparisons were set up, such that the subject modifier of Sentence 1 was paired with each subject in the set of five sentences, the subject of Sentence 1 was paired with each verb in the set of five, the verb of Sentence 1 was paired with each object, the object modifier of Sentence 1 was paired with each object, and the subject of Sentence 1 was paired with each verb-object combination in the set of five. For example, the five concrete sentences in Set 1 were (1) The destructive army pillaged a prosperous village; (2) The colorful snake crushed a screaming monkey; (3) The sharp arrow pierced a frantic bird; (4) The raging elephant trampled an orderly caravan; (5) The falling rock killed a sinful captive. The first ranking comparison would be:

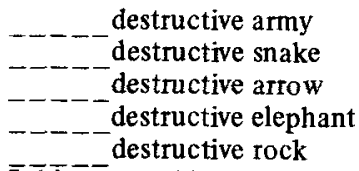

$\bar{S} \overline{\mathrm{b} j \mathrm{j}} \overline{\mathrm{C}} \overline{\mathrm{ts}}$ would be required to rank each of these pairs from the most highly associated (1) to the least highly associated (5). No ties were allowed. This procedure was repeated for the various words in Sentence 2, Sentence 3, Sentence 4, and Sentence 5. Thus, for example, a similar comparison to the above for Sentence 2 would be: colorful army

colorful snake
If some of the cross-sentence pairs were more highly


associated than the pairs used in the initially presented sentences, the absolute rank of the pairs used in the initial sentences would be lower. As there were four groups of sentences and 25 comparisons in each group, there was a total of 200 comparisons for each subject to rank. Concrete and abstract comparisons were presented in alternating order.

The imagery rating scale listed the 20 sentences used in the experiments, alternating concrete and abstract sentences. A five-point scale appeared beneath each sentence, and subjects were instructed to circle a number indicating how "easy it was to form an image of the sentence." On the scale, 1 was described as "very easy to image" and 5 was described as "very difficult to image."

Subjects. Subjects were 60 undergraduate paid volunteers attending classes at Memorial University of Newfoundland. Ten males and 10 females completed each scale. None of the subjects had participated in the earlier experiments.

\section{Results and Discussion}

Table 4 shows the mean concrete and abstract ratings on each of the three scales. For the association rating, the lower the number, the higher the association; thus, as the table indicates, the words in the abstract sentences were rated as more highly associated than the words in the concrete sentences. For the ranking scale, the lower the number, the less interference from high associations between words from different sentences; on this measure, the concrete sentences were less subject to between-sentence association interference than were the abstract sentences. Finally, in the imagery rating, the lower the number, the more easily imaged the sentence; the concrete sentences were rated as considerably more imagable than the abstract sentences.

Analysis of the association ratings were performed by obtaining a mean for all pairs in each sentence and comparing these sentence means on the concrete/abstract dimension. The analysis of variance found a significant difference by subjects $\left[F^{1}(1 / 19)=7.06, p<.025\right]$ but not by sentences. The analysis of the ranking scale was performed by obtaining a mean rank for all pairs in each sentence, and comparing these on the concreteness dimension. The analysis of variance found a significant difference by subjects $\left[F^{1}(1 / 19)=26.41, p<.001\right]$ but not by sentences. The analysis of variance on the imagery scale showed a significant effect due to concreteness $\left[F^{\prime}(1 / 37)=37.81, p<.001\right]$.

Thus there was a significant concrete/abstract difference only on the imagery rating scale. There was a tendency for words used in abstract sentences to be more highly associated and a tendency for concrete sentences to show less between-sentence interferences but neither of these was significant by sentences. In the analyses of the sentence association scores, significance was found in terms of the sentences used in the experiments. Thus it does not seem likely that the significance by subjects with respect to associative relativeness and interference factors (which occurred in
Table 4

Mean Association Rating, Cross-Sentence Interference Rating, and Imagery Rating on the Sentences Used in the Experiments

\begin{tabular}{lccl}
\hline Rating & Concrete & Abstract & $\mathrm{p}$ \\
\hline Association & 3.2 & 2.9 & n.s. \\
Interference & 2.1 & 2.4 & n.s. \\
Imagery & 1.7 & 3.4 & .001 \\
\hline
\end{tabular}

opposite directions) would have had much influence on the results of the previous experiments.

\section{GENERAL DISCUSSION}

The experiments in this paper revealed essentially one main finding - the associational structure of sentences rated high in imagability was easier to remember than the associational structure of sentences rated low in imagability. This result was not explainable in terms of rated comprehension, lexical complexity, semantic association biases, semantic interference between sentences, or limited access to vocabulary items. Significant concrete/abstract differences were found only with respect to the imagery ratings, to the task of organizing the vocabulary items into their previously presented configurations, and to the task of recognizing individual vocabulary items when time was allowed to redintegrate the context in which the word appeared. Significant concrete/abstract differences were not obtained when the decoding period did not allow for the redintegration of the sentence in which the word occurred.

For some reason, the organizational information contained in the abstract sentences was extremely difficult to store. Recently a number of propositional models of verbal memory have been proposed (e.g., Clark \& Chase, 1972; Anderson \& Bower, 1973) which suggest that perceptual information is stored in the same memory system as verbal information and that the underlying associational memory structure has equal access to sentence syntactic information or iconic organizational information. If this were the case, the sentence syntax should have provided the necessary information to form associational connections between elements for both concrete and abstract sentences. As there were no significant concrete/abstract differences in the absolute memory for elemental information (words) and as both types of sentences had identical syntactic structures, it is difficult to explain the significant results with respect to associational information in terms of a semantic propositional model.

These results are more easily explained in terms of an analog model which proposes that there are differences in memory for organizational structure of sentences depending on the ease with which the sentences can be translated into an analog code. Such an assumption is 
made by Paivio's (1971) dual-coding model; it would propose that memory for associational structure in the concrete sentences was superior because they were coded in both the imaginal system (which uses integrative organizational processes) and the verbal system (which uses linguistic organizational processes), whereas the abstract sentences were only coded in the verbal system. However, using this model, it is difficult to explain why the subjects were not significantly superior at recognizing the concrete words when they were' presented individually. If the associational structure was dual coded, it must be assumed that the elements in that structure were also dual coded. Furthermore, the concrete/abstract difference in ability to form images to the individual words was greater at the $3-\mathrm{sec}$ presentation rate, when no significant differences in word recognition were found, than it was at the 6-sec presentation rate, in which there were significant differences in word recognition. Thus imagability had very specific effects on language memory which were not directly predictable from dual coding theory.

They were predictable, however, from a semantic analog model which assumes that both concrete and abstract verbal materials are stored in the same memory system and that this system uses an iconic-based code. Sentences which are easily translated into this iconic-based code (i.e., easily imaged material) are more likely to be stored as one organization. Sentences which are very difficult to represent as a perceptual entity will be more likely represented as several individual memory groupings.

Thus, in Experiment I, subjects asked to comprehend the sentences appeared to judge only the ease with which the individual words in the sentence could be coded; they did not take into account the ease with which these words could be integrated into a holistic structure. The concrete and abstract sentences used in the experiments had equal comprehension ratings. If these raters were also only judging the ease with which the individual words could be encoded, this would explain why there were no significant concrete/abstract differences in the recognition of the individual words when judged at the 3 -sec rate.

It might be argued that understanding a verbal statement requires the formation of a representation in which the separate parts of the statement are formed into a holistic organization. Bransford and Johnson (1973) appear to define comprehension in this way. However, in Experiment I, the subjects did not show that they defined comprehension so. The comprehension group was significantly poorer at remembering the sentence associations but not at remembering the individual words that had appeared in the sentences. Thus for many subjects in this group there had been a memory representation of the individual words but not of the holistic idea.
Surprisingly, even when subjects had practice on the associational memory task they showed no improvement, and they were not significantly longer at encoding the abstract sentences. It appeared as if they did not know how to prepare for the test. Yet it is likely that some improvement could have occurred if subjects had approached the problem correctly. In Experiment I, the elaboration group had a higher mean association score on abstract sentences than any other group given the test (they also showed a larger difference between mean encoding latencies for concrete and abstract sentences than did any other group).

Mistler-Lachman $(1972 ; 1974)$ has shown that when subjects are asked to judge a sentence as meaningful or anomalous, they are much faster at processing and much poorer in recall than subjects asked to integrate the sentence with additional material. She interprets her results in terms of depth of processing: Longer processing times result in a deeper and better memory for the stimuli. However, in Experiment I it was found that the longer processing time resulted in a better integration memory but not in a better memory for the elements which had occurred in the sentences. Thus it may be that the longer a verbal sequence is processed, the more likely it is to be represented as an integrated whole; the deeper and better memory results from this integrative prossessing.

The semantic analog model proposes that the integrative process operates according to iconic principles and that the ease of forming a holistic representation will depend on the ease of representing the sequence as a perceptual whole. Thus, as was found in the present study, sentences which are more easily formed into an image are more easily remembered as a whole.

\section{REFERENCES}

\footnotetext{
Anderson, J. R., \& Bowex, G. H. Human associative memory. Washington, D. C. : Winston \& Sons, 1973.

Begg, I. Recall of meaningful phrases. Journal of Verbal Learning and Verbal Behavior, 1972, 11, 431-439.

Begg, I. Imagery and integration in the recall of words. Canadian Journal of Psychology, 1973, 27, 159-167.

Begg, I., \& Paivio, A. Concreteness and imagery in sentence meaning. Journal of Verbal Learning and Verbal Behavior, 1969,8 , 82

Bower, G.H. Imagery as a relational organizer in associative learning. Journal of Verbal Learning and Verbal Behavior, $1970,9,529+533$.

Bransford, J. D. \& Johnson. M. K. Consideration of some problems of comprehension. In W. G. Chase (Ed.), Visual information processing, New York: Academic Press, 1973.

Clark, H. H. The language-as-fixed-effect fallacy: A critique of language statisties in psychological research. Journal of Verbal Learning and Verbal Behavior, 1973, 12, 335-359.

Clark, H. H., \& Chase, W. G On the process of comparing sentences against pictures. Cognitive Psychology, 1972, 3,
472-517. 472-517.

Holyoak, K. J. The role of imagery in the evaluation of sentences: Imagery or semantic factors? Joumal of Verbal Learning and Verbal Behavior, 1974, 13, 163-166.

Johnson, M. K., Bransford, J. D., Nyberg, S. E., \& Cleary, J. J. Comprehension factors in interpreting memory for abstract and concrete sentences. Journal of Verbal Learning and
Verbal Behavior, 1972, 11,451-454.
} 
Mistler-Lachman, J. Levels of comprehension in processing of normal and ambiguous sentences. Journal of Verbal Learning and Verbal Behavior, 1972, 11, 614-623.

Mistler-Lachman, J. L. Depth of comprehension and sentence memory. Journal of Verbal Learning and Verbal Behavior, $1974,13,98-106$.

Moeser, S. D. Memory for meaning and wording in concrete and abstract sentences. Joumal of Verbal Learning and Verbal Behavior, 1974, 13, 682-697.

Paivio, A. Latency of verbal associations and imagery to noun stimuli as a function of abstractness and generality. Canadian Journal of Psychology, 1966, 20, 378-387.
Paivio, A. Imagery and verbal processes. New York: Holt, Rinehart \& Winston, 1971

Peterson, M. J., \& Murray, A. The enhancement of items and associations. Journal of Experimental Psychology, 1973, 101, 82-89.
(Received for publication August 15, 1974; revision accepted October $31,1974$. ) 